\section{Factores de riesgo para la transmisión de leishmaniasis cutánea en niños de 0 a 5 años en un área endémica de Leishmania (Viannia) braziliensis}

\author{
Risk factors for cutaneous leishmaniasis \\ transmission in children aged 0 to 5 years in an \\ endemic area of Leishmania (Viannia) braziliensis
}

Julia Ampuero 1

Margarita Urdaneta 2

Vanize de Oliveira Macêdo 1

\footnotetext{
1 Núcleo de Medicina Tropical e Nutrição, Universidade de Brasília, Brasília, Brasil.

2 Secretaria de Vigilância em Saúde, Ministério da Saúde, Brasília, Brasil.

Correspondencia

J. Ampuero

Núcleo de Medicina Tropical e Nutrição, Universidade de Brasília. Urbanización Tupac Amaru, Edificio Quinua 402, San Luis, Lima 30, Perú. ausangat@terra.com.pe ausangat@hotmail.com
}

\begin{abstract}
With the purpose of identifying risk factors for cutaneous leishmaniasis transmission in children from 0 to 5 years, a matched case-control study was carried out in Corte de Pedra, Bahia, Brazil, an endemic area of Leishmania (Viannia) braziliensis. Children with a positive leishmanin skin test and one or more active lesions or scars consistent with cutaneous leishmaniasis were defined as cases. Forty cases and 71 controls were selected and matched by age and place of residence. The presence of a family member with a history of cutaneous leishmaniasis in the year prior to the appearance of the disease in the child was found to be an important risk factor $\left(M \hat{O} R_{M H}=17.75\right.$; 95\%CI: 4.08-77.25). No evidence of association between the disease and other risk factors was found, such as child's habits inside or outside the house, domiciliary or peridomiciliary characteristics, or presence of vectors or probable reservoir animals. These findings support the hypothesis that humans serve as both the reservoir and source of infection for this age group.
\end{abstract}

Risk Factors; Cutaneous Leishmaniasis; Leishmania (Viannia) braziliensis

\section{Introducción}

La leishmaniasis tegumentaria americana (LTA) es una enfermedad de alta prevalencia en muchas áreas tropicales y subtropicales del continente. Según la Organización Mundial de la Salud (OMS) se estima que el 90,0\% de los casos de leishmaniasis muco cutánea se presentan en Brasil, Bolivia y Perú, y el 90,0\% de los casos de leishmaniasis cutánea se presentan en Afganistán, Brasil, Irán, Perú, Arabia Saudita y Siria 1. La LTA se presenta en 22 países de América, extendiéndose desde el sur de los Estados Unidos hasta el norte de Argentina 2, donde se han descrito diez especies de Leishmania que pueden producir comprometimiento cutáneo 3 . Esta enfermedad constituye un grave problema de salud pública por los altos costos que representa en la esfera psicológica, social, cultural y económica tanto para el propio paciente, su entorno familiar y los respectivos gobiernos. Todos estos aspectos son de gran impacto para que la leishmaniasis, conjuntamente con la tripanosomiasis africana y el dengue, sean consideradas dentro de la categoría 1 por la OMS para la investigación de nuevos métodos de prevención, diagnóstico y tratamiento, a través del programa de Investigación y Entrenamiento en Enfermedades Tropicales 4 .

Entre 1985 y 1999 fueron notificados al Ministerio de Salud de Brasil 388.155 casos nuevos de LTA. Al final de la década de los 90, to- 
dos los Estados del país registraron casos de leishmaniasis con excepción de Río Grande do Sul. Durante 1999 fueron registrados un total de 30.550 casos, y el Estado de Bahía registró 3.342 casos, con un coeficiente de detección de 25,72 por 100 mil habitantes, ocupando el tercer lugar entre todos los estados, lo que refleja la gravedad que representa la leishmaniasis en este Estado 5.

En Brasil, seis especies de Leishmania son hasta ahora conocidas como causantes de LTA: Leishmania (Leishmania) amazonensis, L. (Viannia) braziliensis, L. (V.) guyanensis, L. (V.) lainsoni, $L$. (V.) shawi y $L$. (V.) naiffi 5 . En el sudeste del Estado de Bahía, en las áreas de Três Braços y Corte de Pedra, fueron descritas dos especies de leishmania: $L$. (V.) braziliensis, responsable por más del 95,0\% de los casos, y L. (L.) amazonensis de presentación más rara 6 .

Los estudios para determinar los factores de riesgo para adquirir LTA han estado dirigidos principalmente a jóvenes y adultos, tanto en Brasil como en otros países de América. Las investigaciones desarrolladas han demostrado que la leishmaniasis está asociada principalmente, al sexo masculino en edad productiva, a actividades agrícolas, extracción de madera, recolección de castañas, caza, pesca, trabajo en lavaderos de oro, actividades de investigación en la floresta tropical entre otros, por lo que ha sido considerada como una enfermedad ocupacional 1,3,7,8. Sin embargo, en América Latina desde inicios de la década pasada, se ha descrito un incremento del número total de casos, una distribución por grupos de edad más amplia, acometimiento semejante entre hombres y mujeres, y casos de familias enteras con la enfermedad que muestran un patrón de transmisión intra y peridomiciliar. Se postula que se ha producido una domesticación gradual de la transmisión a través del tiempo, especialmente en áreas con una larga historia de colonización donde existe poca floresta primaria remanente, ha existido una adaptabilidad de los diferentes componentes del ciclo, y probablemente la presencia de nuevos reservorios secundarios lo que facilitaría este tipo de transmisión 2 .

El comportamiento de la enfermedad en niños es poco conocido existiendo literatura relacionada muy restringida y las existentes están principalmente dirigidas a las características clínicas: relato de casos y revisión de historias clínicas hospitalarias 9,10,11,12,13,14. Otro factor que no permite unificar la información para este grupo de edad es la falta de estandarización de los rangos de edad para ser considerados como niños o adolescentes, y en los estudios poblacionales se hacen referencia a décadas: 0-9, 10-19 7,15, siendo pocos los investigadores que estratificaron los grupos de edad en intervalos menores: 0-5, 6-10, 11-15 años 16; 0-2, 3-7, 8-20 años 17; y 0-1, 1-5 y 5-15 años 14 . Al ser considerados rangos amplios de edad no se puede conocer con profundidad las características inherentes de los grupos menores.

Aproximadamente uno de cada diez casos atendidos en el Centro de Salud de Corte de Pedra entre 1987 y 1995 fueron niños entre 0 y 5 años, con lesiones localizadas predominantemente encima de la cintura, y uno de cada tres presentaba lesiones múltiples 18. Estas características clínicas están asociadas a un mayor riesgo para el desarrollo de lesiones mucosas 19; además estos niños disponen de mayor tiempo durante su vida para presentar un compromiso mucoso. Existe también dificultad para la administración de los tratamientos convencionales, por la toxicidad de los medicamentos, las vías de administración parenteral, y la falta de respuesta terapéutica mencionada por algunos autores 20 . Ante la poca información sobre los factores condicionantes para la transmisión de la LTA en niños, nuestro objetivo fue identificar y evaluar los diferentes factores de riesgo asociados a la presencia de leishmaniasis cutánea en niños de 0 a 5 años que habitaban en el área endémica de Corte de Pedra.

\section{Materiales y métodos}

El estudio fue desarrollado en el área de influencia geográfica del Centro de Salud de Corte de Pedra, establecimiento que funciona como centro de referencia para los pacientes con leishmaniasis de la región bajo la responsabilidad del Núcleo de Medicina Tropical de la Universidad de Brasilia. El Centro de Salud de Corte de Pedra está localizado en el distrito de Corte de Pedra, que pertenece al Municipio Presidente Tancredo Neves creado en 1989; se encuentra localizado a la margen izquierda y derecha de la autopista BR101, en el litoral sur del Estado de Bahía, Brasil, a una distancia aproximada de $275 \mathrm{~km}$ de la ciudad de Salvador, capital del Estado. Ubicado entre 600 y 800 metros sobre el nivel del mar está cubierto principalmente por floresta secundaria en diferentes fases de evolución, producto de una deforestación del área desde hace más de cincuenta años. Los cultivos agrícolas principales de esta región son cacao, claveros (clavo de especia), guaraná, bananas y mandioca. El clima es tropical húmedo con una humedad relativa aproximada de $78,0 \%$ y una temperatura que oscila entre los 16 a $37^{\circ} \mathrm{C}$. Durante 1995 se presentó una pre- 
cipitación anual de $1.161 \mathrm{~mm}$, con una media mensual de 96,75mm (Datos pluviométricos de 1984 a 1995 - mensual/anual/media mensual, suministrados por la Comisión Ejecutiva del Plan de Labranza del Cacao). La población estimada para el municipio sobre la base de los censos realizados por el Instituto Brasileño de Geografía y Estadística en 1980 y 1991, para julio de 1995 fue de 20.275 habitantes, de los cuales 3.664 correspondían a niños entre 0 y 5 años. Aproximadamente el $88,0 \%$ de esta población residía en la zona rural (datos suministrados por la Secretaría de Educación y Cultura, Prefectura Municipal Presidente Tancredo Neves). Entre 1987 y 1995 fueron atendidos un total de 4.275 casos nuevos de leishmaniasis en el Centro de Salud de Corte de Pedra, de los cuáles el 11,5\% correspondían a niños entre 0 y 5 años de edad 18.

Fue realizado un estudio caso-control pareado por edad y área de procedencia, en una razón de un caso para dos controles. Considerando una odds ratio de 4 y un porcentaje de exposición entre los controles de 15,0\%, el tamaño de la muestra fue definido en cuarenta casos y ochenta controles, con un nivel de significancia de $95 \%$ y poder de $80 \%$. Para los casos menores de dos años se seleccionaron controles con \pm seis meses, y para los mayores de dos años fueron seleccionados controles con \pm un año. Esta división se debió al hecho que los niños menores de dos años presentan hábitos más o menos homogéneos como la permanencia de un mayor tiempo dentro de casa o en el peridomicilio próximo 17. En cuanto al área de procedencia se estableció una distancia máxima de $1 \mathrm{~km}$ entre la vivienda de un caso y su control. Todos los pacientes-caso y los controles fueron niños nacidos o residentes en el área durante mínimo un año antes del inicio del estudio.

Fue definido como caso de leishmaniasis cutánea, aquel paciente que presentaba en el examen físico una o más lesiones clínicas activas, o cicatrices compatibles con leishmaniasis, más la prueba de la leishmanina positiva. Los controles fueron caracterizados por la ausencia de lesiones activas evidentes o, antecedente de leishmaniasis, y la prueba de leishmanina negativa.

En una primera etapa de selección fueron identificados todos los niños con edades entre 0 y 5 años ( 0 y 71 meses) que presentaban lesiones compatibles con leishmaniasis cutánea activa, o cicatricial, que buscaron atención de manera espontánea en el Centro de Salud de Corte de Pedra entre junio de 1994 a agosto de 1995 (casos probables prevalentes), y niños que procuraban atención entre septiembre y diciembre de 1995 (casos probables incidentes), todos ellos residentes en un radio no mayor a $100 \mathrm{~km}$ del Centro de Salud de Corte de Pedra. Para la ubicación de los casos prevalentes fueron seleccionadas todas las histórias clínicas del archivo del Centro de Salud de Corte de Pedra de los niños que cumplían con los criterios de inclusión. Los casos incidentes fueron captados en el momento de la consulta. Una vez identificados los casos probables fue localizada la residencia y seleccionados los posibles domicilios donde residían los controles.

Después de la explicación de los objetivos del estudio y la obtención del término de consentimiento firmado por el responsable de cada niño visitado, fue aplicado un cuestionario clínico epidemiológico que fue previamente estandarizado y validado en un estudio piloto en los niños que buscaron atención en el Centro de Salud de Corte de Pedra antes del inicio del estudio. Cada cuestionario tenía una duración aproximada de veinte a treinta minutos y fue aplicado por una sola persona en el domicilio de cada niño previamente identificado como caso probable. La madre fue la persona responsable de responder las preguntas y otras fueron verificadas de forma directa por la entrevistadora como: uso de mosquiteros, presencia de lesiones en animales domésticos, plantíos, tipo de vegetación alrededor de la casa y la evaluación de las lesiones activas o cicatrizales compatibles con leishmaniasis en todos los miembros de la familia.

Terminada la aplicación del cuestionario, se realizó la prueba de la leishmanina a todos los integrantes de la familia entrevistada para permitir la definición de los casos y controles, así como para identificar a otros miembros de la familia con antecedente de exposición a leishmaniasis. La prueba de la leishmanina fue administrada por un técnico entrenado de la Fundación Nacional de Salud, utilizando leishmanina proveniente de un mismo lote, preparada, testada y entregada por el Laboratorio de Inmunología del Hospital Universitario Profesor Edgar Santos de la Universidad Federal de Bahía. El antígeno utilizado fue producido a partir de promastigotes de Leishmania (Leishmania) amazonensis (MHOM/BR/BA-125), con una concentración de $5 \times 10^{6}$ promastigotes $/ \mathrm{ml}$ equivalente a 250 microgramos de $\mathrm{N} / \mathrm{ml}$. El antígeno fue almacenado entre $2-8^{\circ} \mathrm{C}$, y transportado al campo en cajas térmicas con hielo. Fue aplicado en forma intradérmica $0,1 \mathrm{ml}$ de leishmanina en el tercio medio anterior del antebrazo izquierdo, la lectura fue realizada después de 48 a 72 horas, utilizando el método de 
la esferográfica con una única regla estandarizada 21 . Fueron consideradas positivas las reacciones iguales o mayores a $5 \mathrm{~mm}$ de diámetro. No se realizó esta prueba en personas que tenían un resultado registrado en las historias clínicas del Centro de Salud de Corte de Pedra durante los últimos cinco años 7. Fueron excluidos del estudio los niños portadores de enfermedades crónicas severas diagnosticadas clínicamente, tales como: tuberculosis, desnutrición moderada a severa, anemia grave e inmunodepresión.

Fueron evaluadas las siguientes variables relacionadas a los hábitos del niño: uso de mosquiteros, frecuencia de acudir a las plantaciones, tipo de ropa que utiliza frecuentemente tanto de día como de noche, horarios y lugar de baño, lugar de disposición de excretas, actividades diurnas, actividades después de las 18 horas y actividad principal de la madre.

Las variables relacionadas a la presencia de probables reservorios en el domicilio o en el peridomicilio próximo correspondieron al antecedente de leishmaniasis en otros miembros de la familia durante el último año, los cuatro últimos años, y más de cinco años; número de integrantes con prueba de leishmanina positiva; presencia de animales domésticos con lesiones compatibles con leishmaniasis; y el antecedente de leishmaniasis en el vecindario durante el último año.

Las variables relacionadas con el medio ambiente fueron divididas en tres categorías: (1) el micro-ambiente-vivienda correspondiente al lugar donde permanece el niño entre el 50,0 al $100,0 \%$ de su tiempo, recolectándose datos de la localización de la residencia, tipo de material de construcción, cantidad de habitaciones, número de habitantes, fumigación con insecticidas durante el último año, utilización de productos tradicionales con efecto insecticida o repelente para insectos; (2) el área del peridomicilio próximo que fue definido como el espacio comprendido hasta un radio de $10 \mathrm{~m}$ de la casa 8 ; y (3) el peridomicilio macro, aquel espacio situado hasta un radio de $1 \mathrm{~km}$, verificándose la presencia de vegetación en el peridomicilio próximo, tipo de vegetación existente y deforestación durante el último año antes de la aparición de la enfermedad en el niño.

Para la entrada y consistencia de los datos se utilizó el programa Epi Info 6 versión 6.04, y para el análisis estadístico fue utilizado SPSS para Windows versión 10.0. Fueron determinadas las frecuencias de los diferentes aspectos evaluados en los casos y controles y comparadas por medio de testes de chi cuadrado de Pearson $\left(\chi^{2}\right)$ para las variables categóricas y para la comparación de medias fue utilizado el análisis de varianza (ANOVA). Para medir la magnitud de la asociación entre la presencia de leishmaniasis y los factores de riesgo fue calculado el odds ratio pareado para cada variable estudiada utilizando el método de Mantel-Haenszel (MÔR $\mathrm{MH}_{\mathrm{H}}$ ), y un intervalo de confianza de 95\% (IC95\%). Para controlar el efecto de posibles factores confundidores fue realizado un análisis multivariado por medio de regresión logística obteniéndose valores ajustados del odds ratio para los varios factores de riesgo evaluados.

El estudio tuvo en consideración los aspectos éticos de la investigación en seres humanos contenidos en la declaración de Helsinki y considerados en la Resolución del Consejo Nacional de Salud de Brasil y fue aprobado por el Comité de Ética en Investigaciones de la Universidad de Brasilia.

\section{Resultados}

Fueron estudiadas un total de 580 personas pertenecientes a 102 familias, entre casos, controles y el grupo familiar de los hogares inicialmente identificados como posibles participantes del estudio. Fue aplicada la prueba de la leishmanina a un total de 535 personas pues 45 integrantes de estos núcleos familiares ya presentaban resultados registrados en el Centro de Salud de Corte de Pedra durante los últimos cinco años. La edad media de las personas entrevistadas fue de 16,2 $\pm 14,7$ años, con un rango de 4 meses a 73 años. Un 33,1\% (192) eran menores de 6 años, 27,8\% (161) con edades entre 6 a 15 años, y 39,1\% (227) eran mayores de 15 años de edad. En cuanto al sexo, 49,5\% (287) eran del sexo masculino y $50,5 \%$ (293) del sexo femenino.

Del total de entrevistados (580), 49 personas refirieron la presencia de cicatrices compatibles con leishmaniasis antes de 1990 (8,4\%), 17 (2,9\%) entre 1990 y 1994, y 72 personas $(12,4 \%)$ presentaron estas lesiones durante el último año incluyendo los casos del estudio. Ocho de los 138 pacientes con antecedentes de leishmaniasis fueron leishmanina negativos $(5,8 \%), y$ 43 de las 442 personas sin ningún antecedente de la enfermedad o presencia de lesión actual presentaron una prueba de leishmanina positiva $(9,7 \%)$.

Del total de probables casos visitados, fue excluido un caso compatible con leishmaniasis que presentó una prueba de leishmanina negativa y sus respectivos controles; así como una familia donde el control presentó leishma- 
nina positiva, 9 casos no contaron con 2 controles, teniendo finalmente para el análisis 40 casos y 71 controles. No se presentaron diferencias estadísticamente significativas entre casos y controles en cuanto a las variables edad y sexo, siendo ambos grupos comparables (Tabla 1).

El análisis univariado de los factores de riesgo relacionados con los hábitos del niño evidenció que pocos niños utilizaban mosquiteros como medio de protección, sólo tres casos y cinco controles (Tabla 2). No se encontró asociación para factores como: hábito de ir a los plantíos junto a la madre; tipo de ropa utilizada durante la noche; lugar de baño; y las actividades que desarrolla el niño entre las 18 y las 20 horas, horarios de mayor actividad de los flebotomíneos en este área 22,23. Fue encontrado que el hábito de usar poca ropa durante el día está asociado a la presencia de leishmaniasis en este grupo de edad $\left(\mathrm{MÔR}_{\mathrm{MH}}=4,08\right.$; IC95\%: 1,38-12,12).

La presencia de plantaciones de cacao, banana, entre otros a menos de $10 \mathrm{~m}$ de distancia de las casas, que facilita la presencia de flebotomíneos tanto en el peridomicilio próximo como en el intradomicilio; el tipo de vegetación; y la deforestación en el peridomicilio próximo no constituyeron factores de riesgo para la presencia de leishmaniasis en niños. Por otro lado, existe poco hábito de fumigar las casas con insecticidas, sólo ocho familias utilizaban este medio de protección; la mayoría de familias utilizaban medios tradicionales como el quemado de plantas o gomas, los cuales no demostraron ser un factor de protección importante contra la leishmaniasis.

La presencia de uno o más casos de leishmaniasis en la familia durante el último año antes de la aparición de la leishmaniasis en el niño constituyó un factor de riesgo importante para la presencia de esta enfermedad $\left(\mathrm{MÔR}_{\mathrm{MH}}\right.$ = 17,75; IC95\%: 4,08-77,25). No se encontró asociación entre los antecedentes de leishmaniasis mayor a un año dentro de la familia y la presencia de leishmaniasis en el niño, así como la presencia de casos de leishmaniasis en la vecindad (Tabla 3 ).

Del total de personas entrevistadas que refirieron haber tenido una lesión compatible con leishmaniasis (138), 8 presentaron la prueba negativa. De los que refirieron no haber tenido lesiones (442), 43 personas presentaron la prueba positiva. En la presencia de este contexto se verificó en cuantos domicilios de los casos y de los controles había miembros de la familia que cumplían los criterios de leishmaniasis (lesión activa o cicatriz compatible y prueba de leishmanina positiva), posteriormente, los datos
Tabla 1

Características generales de los niños menores de seis años de edad incluidos en el estudio caso-control para determinación de factores de riesgo para leishmaniasis cutánea. Corte de Pedra, Bahía, Brasil, 1994-1995.

\begin{tabular}{|c|c|c|c|}
\hline & $\begin{array}{l}\text { Casos } \\
(n=40)\end{array}$ & $\begin{array}{c}\text { Controles } \\
(n=71)\end{array}$ & $\begin{array}{c}\text { Total } \\
(n=111)\end{array}$ \\
\hline \multicolumn{4}{|l|}{ Sexo* } \\
\hline \multicolumn{4}{|l|}{ Masculino } \\
\hline - $\mathrm{n}$ & 23 & 30 & 53 \\
\hline$\bullet \%$ & 57,5 & 42,3 & 47,7 \\
\hline \multicolumn{4}{|l|}{ Femenino } \\
\hline$\bullet \mathrm{n}$ & 17 & 41 & 58 \\
\hline$\bullet \%$ & 42,5 & 57,7 & 52,3 \\
\hline \multicolumn{4}{|l|}{ Edad (en meses) ${ }^{\star \star}$} \\
\hline Media & 38,0 & 37,7 & 37,8 \\
\hline Desviación estándar & 16,3 & 16,3 & 16,3 \\
\hline Edad mínima & 5 & 6 & 5 \\
\hline Edad máxima & 66 & 70 & 70 \\
\hline
\end{tabular}

fueron organizados según la presencia o ausencia de estas dos condiciones en algún miembro de la familia y estratificados por los períodos de tiempo del antecedente de la lesión (Tabla 4). En este análisis se observa que la presencia en el domicilio de por lo menos una persona con antecedente de leishmaniasis (cicatriz y prueba de leishmanina positiva) en el último año, constituye un factor de riesgo importante para la presencia de leishmaniasis en los niños $\left(\mathrm{MÔR}_{\mathrm{MH}}=11,50\right.$; IC95\%: 3,38-39,19). En contraposición, niños que no tienen familiares en casa con la prueba de leishmanina positiva no tienen riesgo de presentar leishmaniasis $\left(\mathrm{MÔR}_{\mathrm{MH}}=\right.$ 0,22; IC95\%: 0,06-0,81). En el análisis multivariado la presencia de familiares con leishmaniasis en el último año permaneció asociada, de forma independiente, con la enfermedad en los niños (OR = 12,4; IC95\%: 3,60-42,40).

\section{Discusión}

La presencia de casos de leishmaniasis en la familia confirmado con una prueba de leishmanina positiva durante el último año antes de presentarse el caso de leishmaniasis en el niño fue el factor de riesgo más importante encontrado en este estudio. Estos hallazgos, sugieren la probabilidad que el hombre puede actuar como reservorio intradomiciliar del protozoario. La presencia de un caso de leishmaniasis 
Factores de riesgo para leishmaniasis cutánea en niños menores de seis años de edad relacionados con los hábitos del niño. Estudio caso-control pareado, Corte de Pedra, Bahía, Brasil, 1994-1995.

\begin{tabular}{|c|c|c|c|c|c|}
\hline & $\begin{array}{l}\text { Casos } \\
(n=40)\end{array}$ & $\begin{array}{c}\text { Controles } \\
(n=71)\end{array}$ & MÔR $_{\mathrm{MH}^{*}}$ & IC95\% & Valor de $p$ \\
\hline \multicolumn{6}{|l|}{ Uso de mosquitero } \\
\hline No & 37 & 66 & 0,75 & $0,13-4,49$ & 0,87 \\
\hline Sí & 3 & 5 & & & \\
\hline \multicolumn{6}{|c|}{ Acompañar a los plantíos ${ }^{\star \star}$} \\
\hline Sí & 21 & 36 & 1,19 & $0,48-2,94$ & 0,88 \\
\hline No & 16 & 31 & & & \\
\hline \multicolumn{6}{|l|}{ 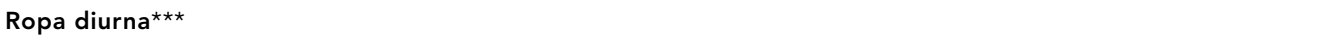 } \\
\hline Cubierto $\leq 50 \%$ & 30 & 36 & 4,08 & $1,38-12,12$ & 0,01 \\
\hline Cubierto $>50 \%$ & 10 & 35 & & & \\
\hline \multicolumn{6}{|l|}{ Ropa nocturna $a^{\star \star \star}$} \\
\hline Cubierto $\leq 50 \%$ & 9 & 17 & 0,85 & $0,32-2,27$ & 0,93 \\
\hline Cubierto $>50 \%$ & 31 & 54 & & & \\
\hline \multicolumn{6}{|l|}{ Lugar de baño } \\
\hline Peri-domicilio & 13 & 13 & 2,67 & $0,89-8,02$ & 0,11 \\
\hline Intra-domicilio & 27 & 58 & & & \\
\hline \multicolumn{6}{|c|}{ Actividad entre $18-20$ horas $^{\star \star \star \star}$} \\
\hline Peri-domicilio & 11 & 11 & 3,13 & $0,86-11,31$ & 0,16 \\
\hline Intra-domicilio & 29 & 60 & & & \\
\hline
\end{tabular}

* $\mathrm{MÔR}_{\mathrm{MH}}=$ estimativa del odds ratio pareado de Mantel-Haenszel; ** se excluyen tres casos y cuatro controles sin datos; ${ }^{\star \star \star}$ hábito de uso de ropa: cubierto $>50 \%$ (utiliza camiseta y pantalón, vestido, camiseta + bermuda), cubierto $\leq 50 \%$ (sólo bermuda, short o sin ropa); ${ }^{\star \star \star \star}$ actividad principal del niño después de las 18 horas: intradomicilio $=$ jugar en casa, dormir y para menores de un año; peri-domicilio = actividades recreativas fuera de casa.

en el intradomicilio podría servir como fuente de infección para los flebotomíneos que ya se encuentran bien adaptados en este medio, como es el caso de Lutzomyia (Nyssomyia) withmani especie predominante en la zona 18,22,23, este posteriormente infectarían a un otro miembro de la familia, especialmente niños de corta edad que desarrollan la mayoría de sus actividades en el intra o en el peridomicilio próximo, hipótesis que ya fue levantada por otros investigadores 24,25 .

Los hechos que apoyarían la hipótesis que el ser humano se podría comportar como un probable reservorio se inician con una publicación de 1927 con el hallazgo de leishmanias a partir de triturados de suspensión de flebotomíneos (L. intermedia) alimentados de los bordes de úlceras de pacientes con leishmaniasis 26, también los experimentos de Rojas \& Scorza 27 en Venezuela que consiguieron infectar ejemplares de L. youngi a través de la alimentación en los bordes de las lesiones de ocho pacientes con leishmaniasis cutánea producida por $L$. (V.) braziliensis. Por otro lado, también se consiguió aislar leishmanias a partir de una lesión cicatrizal de nueve años de evolución 28 , y este mismo investigador encontró en las cicatrices de 16 pacientes $(n=18)$ la prueba del PCR positiva 29 , estos hallazgos también apuntarían a la posibilidad de que personas con lesiones antiguas también podrían constituirse en reservorios de la enfermedad, aunque nosotros no pudimos confirmar este hecho.

El uso de mosquiteros por los niños de Corte de Pedra fue un hábito poco frecuente $(7,2 \%)$; esta baja frecuencia de uso tanto por los casos y los controles no permitió establecer que este hecho constituye un factor de riesgo para desarrollar la enfermedad. Tampoco se pudo comprobar en el presente estudio una asociación entre la presencia de la enfermedad en los niños y el hábito de acompañar a la madre a los plantíos o bosques, a pesar que este factor ha sido relatado como de riesgo importante para 
Factores de riesgo para leishmaniasis cutánea en niños menores de 6 años de edad relacionados con posibles reservorios humanos. Estudio caso-control pareado. Corte de Pedra, Bahía, Brasil, 1994-1995.

\begin{tabular}{|c|c|c|c|c|c|}
\hline & $\begin{array}{l}\text { Casos } \\
(n=40)\end{array}$ & $\begin{array}{l}\text { Controles } \\
(n=71)\end{array}$ & MÔR $_{\mathrm{MH}}{ }^{*}$ & IC95\% & Valor de $p$ \\
\hline \multicolumn{6}{|c|}{$\begin{array}{l}\text { Antecedente de leishmaniasis } \\
\text { en la familia durante el último año }\end{array}$} \\
\hline Sí & 22 & 6 & 17,75 & $4,08-77,25$ & $<0,01$ \\
\hline No & 18 & 65 & & & \\
\hline \multicolumn{6}{|c|}{$\begin{array}{l}\text { Antecedente de leishmaniasis } \\
\text { en la familia entre 1990-1994 }\end{array}$} \\
\hline Sí & 6 & 8 & 1,36 & $0,42-4,37$ & 0,85 \\
\hline No & 34 & 63 & & & \\
\hline \multicolumn{6}{|c|}{$\begin{array}{l}\text { Antecedente de leishmaniasis } \\
\text { en la familia mayor de cinco años }\end{array}$} \\
\hline Sí & 22 & 25 & 2,38 & $0,98-5,79$ & 0,06 \\
\hline No & 18 & 46 & & & \\
\hline \multicolumn{6}{|c|}{$\begin{array}{l}\text { Leishmaniasis en la vecindad } \\
\text { durante el último año }\end{array}$} \\
\hline Sí & 22 & 39 & 1,03 & $0,44-2,43$ & 0,89 \\
\hline No & 18 & 32 & & & \\
\hline
\end{tabular}

* $\mathrm{MÔR}_{\mathrm{MH}}=$ estimativa del odds ratio pareado de Mantel-Haenszel.

las poblaciones adultas 8,30 . La cantidad de ropa que frecuentemente utilizaba el niño durante el día demostró estar asociado a la presencia de leishmaniasis cutánea, es decir, el utilizar poco vestuario concurre para la presencia de una mayor área corporal expuesta para el ataque de los flebotomíneos. La cantidad de ropa utilizada durante la noche no demostró asociación con la presencia de leishmaniasis, la mayoría de madres cubren bien el cuerpo de los niños para dormir, utilizando camisetas y pantalones largos, sin embargo, quedan desprotegidos el área facial y a veces los miembros superiores. Lamentablemente, no encontramos otros estudios dirigidos que nos permitan comparar estos hallazgos.

Algunos autores han señalado la aglomeración humana intradomiciliaria como un factor de riesgo para la transmisión de la leishmaniasis 31,32 . Para el caso del estudio realizado en Corte de Pedra no fue encontrado este hecho como un factor de riesgo. Tampoco la presencia de animales domésticos constituyó un factor de riesgo, a pesar que en Costa Rica se comprobó que la presencia de animales en el intradomicilio y en el peridomicilio ejercerían una atracción sobre los flebotomíneos y así constituirían un factor de riesgo para aquellos niños que tuvieran contacto con estos animales 31 .
No se constató la presencia de animales domésticos con lesiones compatibles con leishmaniasis en las casas visitadas, a pesar que estudios realizados en áreas cercanas (Tres Braços) habían confirmado entre un 3,0 a 9,0\% de infección en perros 33 , y el 16,0\% de infección en jumentos 34 , y se levantaba la posibilidad que estos podrían comportarse como reservorios, hipótesis también mencionada por otros investigadores 35,36; no obstante aún no se tiene evidencia concreta que estos animales puedan comportarse como reservorios 37,38. Con los resultados encontrados en este estudio no podemos afirmar o negar el papel de los animales domésticos en la cadena de transmisión de la leishmaniasis, sin embargo se levanta la posibilidad de que los animales domésticos pueden comportarse como huéspedes accidentales al igual que los seres humanos.

La presencia de plantaciones a menos de $10 \mathrm{~m}$ de la vivienda no demostró ser un factor de riesgo para la presencia de leishmaniasis en niños, hallazgo semejante fue observado por Scorza et al. 17 quienes no consiguieron demostrar una asociación entre transmisión y proximidad (entre 20 a $42 \mathrm{~m}$ ) de los domicilios con las plantaciones. En Corte de Pedra las plantaciones de cacao permanentes crean un microambiente húmedo con poca luz, propicio para el 
Prueba de la leishmanina y antecedentes de lesión en otros miembros de la familia como factor de riesgo para leishmaniasis cutánea en niños menores de seis años de edad. Estudio caso-control pareado. Corte de Pedra, Bahía, Brasil, 1994-1995

\begin{tabular}{|c|c|c|c|c|c|}
\hline & $\begin{array}{l}\text { Casos } \\
(n=40)\end{array}$ & $\begin{array}{l}\text { Controles } \\
(n=71)\end{array}$ & MÔR $_{\mathrm{MH}^{*}}{ }^{*}$ & IC95\% & Valor de $p$ \\
\hline \multicolumn{6}{|c|}{$\begin{array}{l}\text { Todos los integrantes de la familia } \\
\text { son leishmanina (-) }\end{array}$} \\
\hline No & 36 & 48 & 0,22 & $0,06-0,81$ & 0,02 \\
\hline Sí & 4 & 23 & & & \\
\hline \multicolumn{6}{|c|}{$\begin{array}{l}\text { Presencia de al menos un integrante de } \\
\text { la familia con leishmanina }(+) \text { sin cicatriz }\end{array}$} \\
\hline Sí & 3 & 13 & 0,42 & $0,11-1,56$ & 0,28 \\
\hline No & 37 & 58 & & & \\
\hline \multicolumn{6}{|c|}{$\begin{array}{l}\text { Presencia de al menos un integrante } \\
\text { de la familia con leishmanina }(+) \text { y cicatriz } \\
\text { anterior a } 1990\end{array}$} \\
\hline Sí & 10 & 23 & 0,63 & $0,24-1,64$ & 0,47 \\
\hline No & 30 & 48 & & & \\
\hline \multicolumn{6}{|c|}{$\begin{array}{l}\text { Presencia de al menos un integrante } \\
\text { de la familia con leishmanina }(+) \text { y cicatriz } \\
\text { entre 1990-1994 }\end{array}$} \\
\hline Sí & 2 & 6 & 0,58 & $0,11-3,14$ & 0,80 \\
\hline No & 38 & 65 & & & \\
\hline \multicolumn{6}{|c|}{$\begin{array}{l}\text { Presencia de al menos un integrante } \\
\text { de la familia con leishmanina }(+) \text { y lesión } \\
\text { en el último año }\end{array}$} \\
\hline Sí & 21 & 6 & 11,50 & $3,38-39,19$ & $<0,01$ \\
\hline No & 19 & 65 & & & \\
\hline
\end{tabular}

* ${ }^{\mathrm{MOOR}} \mathrm{MH}_{\mathrm{MH}}=$ estimativa del odds ratio pareado de Mantel-Haenszel.

desarrollo de los flebotomíneos cerca o junto a la vivienda 22 , entonces la casa y el peridomicilio próximo constituyen un mismo nicho ecológico, donde los vectores pueden volar libremente entre los dos ambientes transmitiendo la infección si estos se encuentran infectados, la pregunta es $i$ dónde se infectan? ¿En este microambiente? $i \mathrm{O}$ en las florestas remanente localizadas a mayor distancia de las casas o de los lugares de trabajo?

Estos hallazgos aunados a las características clínicas que se presentan en los niños, donde ambos sexos son igualmente afectados, sugieren un patrón de transmisión intra y peridomiciliar en el área de Corte de Pedra, hecho que nos permite proponer medidas de control es- pecíficas dirigidas a estos grupos de edad como los ya propuestos por varios autores: la utilización de mosquiteros o cortinas impregnadas y la utilización de insecticidas con efecto residual en las casas $2,3,39$, y en la perspectiva de que el ser humano puede ser un probable reservorio debe de realizarse una detección y un tratamiento precoz de los casos que se presentan en el área. El potencial uso de las medidas de intervención propuestas para la prevención y control de la leishmaniasis requieren de estudios de evaluación de la efectividad con componentes de promoción de la participación directa de la comunidad en su implementación, para asegurar su sostenibilidad. 


\section{Resumen}

Fue realizado un estudio caso-control pareado en Corte de Pedra, Bahía, Brasil, área endémica de Leishmania (Viannia) braziliensis para evaluar los diferentes factores de riesgo asociados a la presencia de leishmaniasis cutánea en niños de 0 a 5 años. Fueron considerados como casos, los niños con prueba de leishmanina positiva y que presentaban en el examen físico, una o más lesiones clínicas, activas o cicatrizales, compatibles con leishmaniasis cutánea. Fueron seleccionados 40 casos y 71 controles que fueron pareados por edad y área de residencia. La presencia de algún otro miembro de la familia con antecedente de leishmaniasis cutánea durante el año anterior a la aparición de la enfermedad en el niño demostró ser un importante factor de riesgo (MÔR $R_{M H}=17,75$; IC95\%: 4,08-77,25). No se encontraron evidencias de asociación con otros factores, como hábitos del niño dentro y fuera de casa, características de la vivienda y del peridomicilio, presencia de vectores y animales como probables reservorios. Estos hallazgos apoyan la hipótesis que el ser humano podría comportarse como un posible reservorio y servir de fuente de contagio para este grupo de edad.

Factores de Riesgo; Leishmaniasis Cutánea; Leishmania (Viannia) braziliensis

\section{Colaboradores}

J. Ampuero escribió el texto original que fue revisado críticamente por las co-autoras con aporte intelectual relevante en todos los aspectos del trabajo y participación en la discusión de los aspectos apuntados por los revisores, así como en la revisión de cada modificación realizada. J. Ampuero y V. O. Macêdo participaron en el diseño y ejecución del estudio, y en la fase de análisis de los datos, participan J. Ampuero y M. Urdaneta.

\section{Agradecimientos}

Al Prof. Philip Davis Marsden (In memoriam) por la orientación para la realización del presente trabajo, al Prof. Edgar Carvalho (UFBA), al Dr. Gustavo Romero $(\mathrm{UnB})$ por la revisión y sugerencias al presente artículo, y a Edinaldo Lima do Lago por el apoyo para la localización de los pacientes y la aplicación de la prueba de la leishmanina.

\section{Referencias}

1. World Health Organization. Programme for the surveillance and control of leishmaniasis. http:// www.who.int/emc/diseases/leish/leishmaniasis.pdf (accesado el 12/Sep/2004).

2. Desjeux P. The increase in risk factors for leishmaniasis worldwide. Trans R Soc Trop Med Hyg 2001; 95:239-43.

3. Ashford R. The leishmaniases as emerging and reemerging zoonoses. Int J Parasitol 2000; 30:126981.

4. Remme J, Blas E, Chitsulo L, Desjeux P, Engers H, Kanyok T, et al. Strategic emphases for tropical diseases research: a TDR perspective. Trends Parasitol 2002; 18:421-6.

5. Fundação Nacional de Saúde. Manual de controle da leishmaniose tegumentar americana. Brasília: Fundação Nacional de Saúde, Ministério da Saúde; 2000.

6. Rosa A, Cuba C, Vexenat A, Barreto A, Marsden P Predominance of Leishmania braziliensis braziliensis in the regions of Três Braços and Corte de Pedra, Bahia, Brazil. Trans R Soc Trop Med Hyg 1988; 82:409-10.

7. Jones T, Johnson W, Barreto A, Lago E, Badaro R, Cerf B, et al. Epidemiology of American cutaneous leishmaniasis due to Leishmania braziliensis braziliensis. J Infect Dis 1987; 156:73-83.

8. Weigle K, Santrich C, Martinez F, Valderrama L, Saravia N. Epidemiology of cutaneous leishmaniasis in Colombia: environmental and behavioral risk factors for infection, clinical manifestations, and pathogenicity. J Infec Dis 1993; 168:709-14.

9. Nishino L, Ragiotto A, Almeida N, Santamaria J. Leishmaniose em criança. An Bras Dermatol 1986; 61:23-8.

10. Cucé L, Belda Jr. W, Zolli C. Leishmaniose tegumentar americana na infância: aspectos epidemiológicos, clínicos e terapêuticos. An Bras Dermatol 1990; 5a:18S-9S.

11. Carrada-Bravo T. La leishmaniasis en los niños. Progresos recientes. Bol Med Hosp Infant Mex 1984; 41:356-62.

12. Vallejo L, Villalonga J. Leishmaniasis infantil. Rev Arg Dermatol 1985; 66:1-7.

13. Miranda O, Gimenez MF, Waisman R, Gimenez MM. Leishmaniasis infanto-juvenil. Centro Dermatológico - Resistencia - (1975-1986). Rev Arg Dermatol 1988; 69:91-5.

14. Diógenes M, Gonçalves H, Lima J, Menezes R, Gonçalves HM. Leishmanioses na infância: aspectos epidemiológicos, clínicos e terapêuticos (Estado do Ceará-Brasil). An Bras Dermatol 1993; 68:209-12.

15. Llanos-Cuentas E, Marsden P, Lago E, Barreto A Cuba C, Johnson W. Human mucocutaneous leishmaniasis in Três Braços, Bahía - Brazil. An area of Leishmania braziliensis braziliensis transmission. II. Cutaneous disease. Presentation and evolution. Rev Soc Bras Med Trop 1984; 17:169-77.

16. Costa JML, Balby I, Rocha E, Silva A, Rebêlo J, Ferreira L, et al. Estudo comparativo da leishmaniose tegumentar americana em crianças e adolescentes procedentes das áreas endêmicas de $\mathrm{Bu}$ riticupu (Maranhão) e Corte de Pedra (Bahia), 
Brasil. Rev Soc Bras Med Trop 1998; 31:279-88.

17. Scorza J, Valera M, Moreno E, Jaimes R. Encuesta epidemiológica sobre leishmaniasis cutánea. Un estudio en Mérida, Venezuela. Bol Oficina Sanit Panam 1983; 95:118-32.

18. Ampuero J. Fatores de risco para a transmissão de leishmaniose cutânea em crianças de 0 a 5 anos em uma área endêmica de Leishmania (Viannia) braziliensis [Dissertação de Mestrado]. Brasília: Núcleo de Medicina Tropical e Nutrição, Universidade de Brasília; 1996.

19. Llanos-Cuentas A, Marsden P, Cuba C, Barreto A, Campos M. Possible risk factors in development of mucosal lesions in leishmaniasis. Lancet 1984; 2:295.

20. Palacios R, Osorio L, Grajales L, Ochoa M. Treatment failure in children in a randomized clinical trial with 10 and 20 days of meglumine antimonato for cutaneous leishmaniasis due to Leishmania viannia species. Am J Trop Med Hyg 2001; 64:187-93

21. Sokal J. Measurement of delayed skin-test responses. New Engl J Med 1975; 293:501-2.

22. Vexenat J, Barreto A, Cuba C, Marsden P. Características epidemiológicas da leishmaniose tegumentar americana em uma região endêmica do Estado da Bahia. III. Fauna flebotomínica. Mem Inst Oswaldo Cruz 1986; 81:293-301.

23. Sherlock I, Maia H, Dias-Lima A. Resultados preliminares de um projeto sobre a ecologia dos flebotomíneos vetores da leishmaniose tegumentar no estado da Bahia. Rev Soc Bras Med Trop 1996; 29:207-14.

24. Marzochi MCA. Leishmanioses no Brasil. As leishmanioses tegumentares. J Bras Med 1992; 63:82104.

25. Falqueto A. Especificidade alimentar de flebotomíneos em duas áreas endémicas de leishmaniose tegumentar no Estado do Espírito Santo, Brasil. Rev Soc Bras Med Trop 1997; 30:531-2.

26. Aragão H. Leishmaniose tegumentar e sua transmissão pelos phlebotomos. Mem Inst Oswaldo Cruz 1927; 20:177-86.

27. Rojas E, Scorza J. Xenodiagnóstico con Lutzomyia youngi en casos venezolanos de leishmaniasis cutánea por Leishmania braziliensis. Mem Inst Oswaldo Cruz 1989; 84:29-34.

28. Schubach A, Marzochi M, Araújo M, Conceição N. Healed lesion of cutaneous leishmaniasis - a positive culture for Leishmania $s p$. in the scar tissue years after cure. Mem Inst Oswaldo Cruz 1987; 82 Suppl:64.

29. Schubach A, Haddad F, Oliveira-Neto MP, Degrave W, Pirmez C, Grimaldi Jr. G, et al. Detection of Leishmania DNA by polymerase chain reaction in scars of treated human patients. J Infect Dis 1998; 178:911-4.
30. Lepont F, Mouchet J, Desjeux P, Torres J, Richard A. Epidémiologie de la leishmaniose tégumentaire en Bolivie. 2. Modalités de la transmission. Ann Soc Belg Med Trop 1989; 69:307-12.

31. Rojas J. Three research perspectives on transmission related risk factors for cutaneous leishmaniasis in Costa Rica. I. New strategy for the control of cutaneous leishmaniasis: the case of Acosta, Costa Rica. In: Wijeyaratne P, Goodman T, Espinal C, editors. Leishmaniasis control strategies: a critical evaluation of IDRC-supported research Ottawa: Editorial International Development Research Center; 1992. p. 223-9.

32. Yadon Z, Rodriguez L, Davies C, Quigley M. Indoor and peridomestic transmission of American cutaneous leishmaniasis in northwestern Argentina: a retrospective case-control study. Am J Trop Med Hyg 2003; 68:519-26.

33. Barreto A, Cuba C, Vexenat J, Rosa A, Marsden P, Magalhães A. Características epidemiológicas da leishmaniose tegumentar americana em uma região endêmica do Estado da Bahia. II. Leishmaniose canina. Rev Soc Bras Med Trop 1984; 17:59-65.

34. Barreto A, Vexenat J, Rosa A, Cuba C, Lago E, Peterson N. Epidemiological study of cutaneous leishmaniasis in equines from an endemic area of the State of Bahia, Brazil. Mem Inst Oswaldo Cruz 1986; 81 Suppl:63.

35. Falqueto A, Coura J, Barros G, Grimaldi G, Sessa P, Carias V, et al. Participação do cão no ciclo de transmissão da leishmaniose tegumentar no município de Viana, Estado do Espírito Santo, Brasil. Mem Inst Oswaldo Cruz 1986; 81:155-63.

36. Aguilar C, Rangel E, Garcia L, Fernandez E, Momen H, Grimaldi G, et al. Zoonotic cutaneous leishmaniasis due to Leishmania (Viannia) braziliensis associated with domestic animals in Venezuela and Brazil. Mem Inst Oswaldo Cruz 1989; 84:19-28.

37. Reithinger R, Davies C. Is the domestic dog (Canis familiaris) a reservoir host of American cutaneous leishmaniasis? A critical review of the current evidence. Am J Trop Med Hyg 1999; 61:530-41.

38. Davies CR, Reithinger R, Campbell-Lendrum D, Feliciangeli D, Borges R, Rodriguez N. The epidemiology and control of leishmaniasis in Andean countries. Cad Saúde Pública 2000; 16:925-50.

39. Campbell-Lendrum D, Dujardin JP, Martinez E, Feliciangeli D, Perez JE, Silans L, et al. Domestic and peridomestic transmission of American cutaneous leishmaniasis: changing epidemiological patterns present new control opportunities. Mem Inst Oswaldo Cruz 2001; 96:159-62.

Recibido el 06/Ene/2004

Versión final presentada el 17/Sep/2004 Aprobado el 27/Sep/2004 